\title{
Las patentes farmacéuticas en razón de la covid-19 desde la perspectiva del law and economics movement*
}

The Pharmaceutical Patents in Reason of Covid 19 from the Perspective of Law and Economics Movement

Edwin Jesith Bernal Ramirez q.e.p.d.

Universidad Militar Nueva Granada, Colombia

DOI: https://doi.org/10.11144/Javeriana.vj70.pfrc

ORCID: https://orcid.org/0000-0002-2876-7989

David Andrés Aguirre Soriano ${ }^{a}$

Universidad Externado de Colombia, Colombia

Recibido: 01 Septiembre 2020

Aceptado: 10 Febrero 2021

david.aguirre01@est.uexternado.edu.co

Publicado: 22 Octubre 2021

ORCID: https://orcid.org/0000-0001-7779-5815

\section{Resumen:}

Este trabajo tiene por objeto dilucidar el dilema relativo al monopolio explotación creado en virtud de las patentes de invención farmacéuticas, que si bien incentivan la innovación, a su vez, representan obstáculos para el acceso a medicamentos esenciales, por cuanto un bien patentado, por lo general se venderá a altos costos en el mercado. Ello repercute en el disfrute y goce del derecho a la salud de los pacientes, al ser bienes con poca elasticidad o sustitutos cercanos, mientras se encuentre en vigor la patente; ningún otro competidor podrá efectuar sin autorización del titular los actos mencionados en el artículo 28 del Acuerdo sobre los Aspectos de los Derechos de Propiedad Intelectual relacionados con el Comercio.

Palabras clave: patentes, análisis económico del derecho, flexibilidades ADPIC, patentes amplias, patentes estrechas.

Abstract:

This paper have by object to clarify the dilemma related with the exploitation monopoly created by pharmaceutical invention patents, which, while incentive the innovation, in turn, represent obstacles to accessing essential medicines, as a patented thing, and therefore, it will be sold at high costs in the market. This affects the enjoyment of the right to health of patients, because it's products with a few elasticity or near substitutes, and while the patent be valid; No other competitor can carry out, without the authorization of the owner, the acts mentioned in Article 28 of the Trade Related Aspects of Intellectual Property Rights Agreement.

Keywords: patents, economic analysis of the law, TRIPS flexibilities, broad patents, narrow patents.

\section{Introducción}

Los productos farmacéuticos tienen relación directa con intereses superiores en la esfera humana, como es el caso de los derechos fundamentales a la vida, salud, los cuales tienen una directa repercusión en el ámbito personal de los individuos.

Las patentes farmacéuticas se encuentran protegidas como una institución de la propiedad industrial, protegidas a nivel internacional a partir del Convenio de París de 1883. No obstante, cuando se utilizan de manera ineficiente y sin un enfoque dirigido al bien común, y el bienestar de la población se erigen como verdaderos monopolios con negativas incidencias en los precios del producto y en la dificultad del acceso por parte de los consumidores, principalmente en los países en vía desarrollo.

El monopolio temporal ${ }^{1}$ de explotación de las patentes de invención tiene su fundamento legal en los derechos otorgados a su titular y estipulados en el artículo 28 de los ADPIC y a nivel regional en el artículo 52 de la Decisión 486, estos inciden, junto a la infraestructura en salud, las políticas sanitarias, la rigurosidad en el examen de patentabilidad, en el acceso a medicamentos, sobre todo en aquellos países en los cuales

Notas de autor

\footnotetext{
${ }^{a}$ Autor de correspondencia. Correo electrónico: david.aguirre01@est.uexternado.edu.co
} 
su economía se basa en la importación de tecnología, ocasionando enormes costos sociales en el acceso a medicamentos esenciales.

A su vez, con la proliferación de Tratados de Libre Comercio, se han ampliado los derechos de propiedad intelectual aún más que lo establecido en los ADPIC, particularmente algunas legislaciones otorgan un monopolio temporal a los datos de prueba ${ }^{2}$.

Además, en virtud del artículo 2 de la Declaración Universal sobre Bioética y Derechos Humanos, se exhorta a los Estados a brindar atención médica de calidad y acceso a medicamentos esenciales. Como lo señala este tema "debe ser analizado y discutido en el ámbito de políticas sanitarias, ya que de otra manera se cae en la simplificación de dejar al mercado como regulador de la salud colectiva"3.

Además, el Comité\# de Derechos Económicos, Sociales y Culturales de Naciones Unidas en la Observación General n. ${ }^{\circ} 14^{4}$ estableció que los Estados deben garantizar la disponibilidad de medicamentos esenciales.

Por otro lado, en el escenario actual de la crisis sanitaria mundial, en virtud de la pandemia ocasionada por la covid-19, despierta interrogantes y preguntas a esta institución jurídica sobre las herramientas destinadas a incentivar la investigación y el desarrollo de nuevas invenciones que permitan preservar la salud y, por consiguiente, la calidad de vida de la humanidad sin anular los incentivos económicos naturales de los laboratorios farmacéuticos. En consecuencia, resulta pertinente observar el desfile de las patentes de medicamentos por la pasarela de algunos de los postulados del Análisis Económico del Derecho, con miras a ubicar un punto de equilibrio económico que haga posible el acceso universal a las vacunas y que medicamentos la humanidad espera con tanto recelo y la necesidad de mantener a flote esta industria a través de los estímulos que le ofrece la patentabilidad de estas invenciones y descubrimientos.

Ahora bien, desde el Acuerdo sobre los Aspectos de los Derechos de Propiedad Intelectual relacionados con el Comercio (ADPIC) se establecieron un conjunto de flexibilidades que los Estados pueden utilizar para garantizar a su población el acceso a medicamentos; así mismo, en la Declaración de Doha se establecen disposiciones para salvaguardar la salud pública. Podemos destacar las licencias obligatorias que son una limitación al derecho de explotación del titular de la patente de invención, más no son en sentido estricto una expropiación, toda vez que no se cercena la titularidad y se garantiza una compensación a favor del titular. Esta institución busca garantizar el acceso a estas invenciones, de manera que aportan eficiencia económica e incentivos positivos con miras a mantener la inversión en desarrollo investigativo, sin que con esto la propiedad intelectual sobre estas creaciones y descubrimientos científicos se desmarque de la función social y el bien común como criterio orientador y limitante del derecho de propiedad, incluida la titularidad en materia de patentes de medicamentos.

El debate se organiza, presentando algunas consideraciones sobre la visión económica de las patentes farmacéuticas, seguido del análisis económico del paradigma de las patentes de medicamentos respecto de la covid-19 para describir la herramienta concebida como licenciamiento obligatorio como factor de eficiencia económica en este mercado.

\section{Consideraciones sobre las patentes farmacéuticas y su visión económica}

La humanidad ha tenido innumerables avances a nivel tecnológico en virtud de la capacidad de discernimiento, espíritu investigativo y su talento para construir herramientas con el propósito de adaptarse al mundo en el que vive. A su vez, el ser humano posee un nivel intelectual que le permite modificar las cosas existentes incorporándoles algo para un determinado fin: artístico, científico o estético. De esta manera ha trasformado el entorno en el que vive mediante el uso de la tecnología 5 . Al mismo tiempo, cada vez ha utilizado diferentes técnicas que le han permitido domesticar el mundo salvaje e incómodo para pasar a uno más cómodo con la posibilidad de tener un disfrute y goce de las actividades en su vida cotidiana. 
Además, el ser humano ha avanzado progresivamente gracias a la tecnología, desde la fabricación de instrumentos de piedra para cazar animales, el descubrimiento del fuego hace $300.000 \mathrm{años}^{6}$, la invención de la rueda, la implementación de técnicas de agricultura en el año 12.000, constituyen algunos hitos relevantes en la historia de la humanidad ${ }^{7}$.

El progreso de la humanidad en la utilización de la tekné para expresar las obras y elaborar sus herramientas u utensilios de trabajo ${ }^{8}$, no se concedió una protección a las creaciones intelectuales en el derecho antiguo, así en "el derecho romano no existió ni el derecho de autor ni la propiedad industrial. En efecto, las creaciones, cualquiera que fuera su destinación en la sociedad, como los muebles, la cerámica, las esculturas, las pinturas, no se hacía una distinción con respecto al interés artístico que estos objetos podían presentar, eliminando así cualquier distinción entre artista y artesano"'.

Posteriormente, en la Edad Media, los detentadores del poder ${ }^{10}$ otorgaron algunos privilegios concedidos para la explotación de molinos de viento en el 1105. Asimismo, en Bordeaux en el 1250 se le otorgo a Bonafusus de Santa Columbia un privilegio para la fabricación de telas de varios colores ${ }^{11}$ por un periodo de 15 años $^{12}$.

Después, en el 1331, se concedió un privilegio al tejedor flamenco Johann Kempe, otorgado por Eduardo III. En 1474, por medio del Estatuto Parte Veneziana, se otorgó un monopolio de explotación de 10 años, inicialmente en temas de actividades de la industria minera en la región de Tirol en Italia. En 1624, se expidió el Estatuto de Monopolios ${ }^{13}$, el cual constituyó un cambio de paradigma al acabar el arbitrio de los reyes de conceder monopolios de explotación ${ }^{14}$.

En 1787, en Estados Unidos, la Constitución consagró la protección a la ciencia y artes por un tiempo limitado. Más tarde, en 1791, en Francia se creó un sistema de registro de invenciones nacionales e internacionales, mientras que, en Estados Unidos, en 1793, se expidió la Ley de Patentes: "el sistema de patentes añadió el combustible del interés para el fuego del ingenio" ${ }^{15}$. En Francia, mediante la Ley del 18 de marzo de 1806 se concedió una protección a los diseños industriales, la cual era otorgada por le Conseil de prud'hommes ${ }^{16}$. Hasta ese entonces existían formas de proteger las invenciones y obras a nivel nacional, pero no se contaba con instrumentos de armonización a nivel internacional, sino que fue a partir de 1883 que entró en vigencia el Convenio de París para proteger la propiedad industrial, estableciendo mínimos estándares de protección en los países miembros. Hoy en día se resaltan los ADPIC de 1994, la Decisión 486 de la CAN, el Convenio Europeo de Patentes, el Tratado de Cooperación en materia de patente (PCT), la Declaración de Doha como instrumentos regionales e internacionales para la protección de la propiedad industrial.

En lo que concierne a las patentes de invención son una institución de la propiedad industrial que se conceden por intermedio de un trámite administrativo ante una oficina nacional competente, tienen validez territorial en la jurisdicción donde se otorgó la respectiva patente al solicitante, es decir, a quién creó un producto o procedimiento en cualquier campo de la tecnología. Los requisitos, considerados por los ADPIC, en el artículo 27, para el otorgamiento de la patente son la novedad, el nivel inventivo y la aplicación industrial. Otorgan a su titular un monopolio de explotación de 20 años a partir de la solicitud, confiriéndole los derechos señalados en el artículo 28 de los ADPIC.

Por consiguiente, no existe una definición legal de invención, toda vez que el avance del derecho es lento en cuanto a los acontecimientos sociales, tecnológicos y económicos, pero a nivel jurisprudencial se ha establecido "como una idea que constituye una regla técnica, es decir una enseñanza para utilizar metódicamente fuerzas de la naturaleza para obtener un resultado causal y perceptible" ${ }^{17}$.

Respecto a la justificación del sistema de propiedad intelectual, existen diferentes teorías, por ejemplo la personalidad, propiedad y variedad cultural. Según la primera, desarrollada por Hegel, "el individuo manifiesta su voluntad creativa y se apropia de esas obras que imprimen su personalidad al exteriorizar su identidad" ${ }^{18}$, sobre este punto podemos encontrar la primera ley de patentes de Francia de 1791, en la cual 
se mencionó: "Toute découverte ou nouvelle invention est la propriété de son auteur, auquel la loi en garantit la pleine et entière jouissance" [Todo descubrimiento o nueva invención es propiedad de su autor, a quien la ley garantiza el pleno y completo disfrute $]^{19}$. Esta disposición resulta cuestionable hoy en día toda vez que el titular de la patente de invención será la persona que primero presente la solicitud en la oficina nacional competente y no el primer inventor del sistema de premier déposant.

Respecto a la teoría del trabajo elaborada por Locke, según la cual "el individuo se apropia de bienes disponibles transformándolos en algo a través del trabajo, ello le permite excluir a los demás del uso de la modificación efectuada sin su consentimiento" ${ }^{20}$, sobre este punto podemos apreciar que actualmente se le otorga la patente de invención a quien cumpla con los requisitos de patentabilidad, es decir, a quien supere el estado de la técnica y haga un salto cualitativo en la tecnología respectiva y esto no sea obvio para el examinador de la oficina de patentes. En relación con la teoría de la Variedad Cultural, la propiedad intelectual se erige como "una conquista que permite garantizar un bienestar social, difundir de manera libre contenidos que puedan ser comprendidos de dominio público al servicio de la humanidad"21.

En relación con lo anterior, también se justifican las patentes de invención como un incentivo que recibe el inventor, en efecto, "el sistema de patentes lo estimula al promover la explotación de nuevas invenciones y garantizarle al inventor un monopolio de explotación exclusivo para de esta manera obtener ganancias y reinvertir parte de ellas en investigación y desarrollo"22.

Por otra parte, desde el análisis económico del derecho, se destaca la obra de Coase sobre los costos sociales, en la que el autor analizó las acciones de las empresas que pueden causar daños a otras empresas. Dicho estudio permite responder al interrogante: ¿cómo compensar a los individuos por los efectos negativos de las acciones de otros individuos (externalidades)? En efecto, basándose en el control de la contaminación, explica que si existen derechos de propiedad claramente afectados a todos los recursos considerados, y si todos los agentes económicos pueden relacionarse para negociar entre ellos, entonces los propios agentes estarán incentivados a celebrar acuerdos voluntarios para transferir los costos de la contaminación de las víctimas a los responsables. De esta forma, en ausencia de costos de transacción, se puede obtener una asignación óptima de recursos, sin ninguna intervención legal, mediante la libre negociación de los derechos de propiedad. Esto también significa que si, por el contrario, los costos de transacción son altos, entonces el resultado de la negociación ya no será óptimo. Otros arreglos institucionales pueden resultar más efectivos ${ }^{23}$.

Así mismo, existen múltiples críticas del sistema de patentes de invención, respecto al acceso de los medicamentos y la exclusión de la competencia, puesto que "al excluir la competencia los productos pueden resultar más costosos e incluso la patente puede ni ser explotada. Además, la remuneración puede resultar desproporcionada respecto al mérito de la invención" ${ }^{24}$. Hay que destacar que "la tercera parte de la población mundial carece de acceso regular a los medicamentos; el 75\% de la humanidad vive en los países en desarrollo y tan solo compra el $8 \%$ de los productos farmacéuticos que se venden en el mundo" 25 , ello ocasionado por los elevados costos de los medicamentos y el monopolio otorgado a los titulares de las patentes farmacéuticas, que tienen la libertad para fijar los precios en el mercado. Es por esta tensión existente entre el sistema de propiedad intelectual y el sistema internacional de los derechos humanos que resulta imperioso tener en cuenta los intereses de salud al implementar los ADPIC en los países en vía de desarrollo ${ }^{26}$.

En el contexto económico del mundo actual existen diversos espacios estratégicos de discusión, como el Foro Económico Mundial, en el cual se planifican los destinos de la globalización capitalista. Así, en Davos, se puede apreciar la interrelación entre los líderes de las corporaciones, los líderes políticos de los Estados nación dominantes y las instituciones supranacionales, que colaboran mano a mano en sus propósitos ${ }^{27}$.

La enseñanza que nos deja Davos es la siguiente: "ningún mercado puede subsistir sin orden y regulación política" ${ }^{28}$. Los líderes de los Estados nación dominantes y las instituciones supranacionales buscan marcos regulatorios internacionales para favorecer la libre circulación de mercancías; así se concibió la Lex mercatoria, que tiene la capacidad de regular el comercio transfronterizo entre los actores del sistema económico 
internacional. También existen organizaciones internacionales que, en las relaciones multilaterales entre Estados nación, ejercen autoridad y fuerza sobre otros contrincantes, que varía dependiendo de la estrategia ${ }^{29}$. Se creó la Organización Mundial del Comercio (OMC), en la cual los Estados tienden a imponer sus intereses a través de acuerdos comerciales a países en vía de desarrollo.

De la misma manera, el sistema de patentes incrementa la dependencia que tienen los países en vía de desarrollo respecto a aquellos con altos estándares de innovación, investigación y desarrollo. En efecto, los países desarrollados cuentan con un mayor margen de presión en las negociaciones internacionales comerciales, sobre el particular se ha señalado que

si bien es cierto que hoy en día los países en desarrollo tienen mayor acceso a los mercados desarrollados, no poseen discrecionalidad para elegir las reglas comerciales y la violación de estas normas crearía una amenaza de reacciones económicas desfavorables por parte de los países desarrollados. Para asegurar el cumplimiento (enforcement), se optó por el sistema de solución de controversias del GATT, que era más eficaz que los mecanismos de solución de controversias de la OMPI y, además, permitía supervisar y controlar la legislación pertinente de los países adherentes. ${ }^{30}$

En la actualidad, China es el país con más solicitudes de patentes de invención en el mundo 58.990 por el sistema PCT superando a los Estados Unidos con $57.840^{31}$. De esta manera, la hegemonía sigue imponiéndose a nivel mundial con poca transferencia de tecnología hacia los países en vía de desarrollo que son utilizados como receptores de solicitudes de patentes para asegurar la protección de las patentes en diversas jurisdicciones. Además, el actual sistema de patentes requiere de un entramado burocrático, por lo que se requiere fortalecer las capacidades informáticas y bases de datos para agilizar búsquedas.

Por otra parte, han surgido diferentes posturas que planean cambios en el sistema, otorgando patentes a las innovaciones eficaces; así, por ejemplo, India reformó su Ley de Patentes introduciendo la sección $3 \mathrm{~d}$, la cual establece que no se permitirá volver a patentar sales, ésteres, éteres, o alguna otra presentación química nueva de una sustancia, a menos que esta contenga propiedades distintas a las del producto original. Por lo anterior, hubo un litigio con Novartis, que pretendió patentar el medicamento SOFOSBUVIR para tratar la hepatitis $\mathrm{C}$, pero, de acuerdo con esa nueva exigencia, la autoridad nacional de la India negó la patente de invención. Actualmente se permite la fabricación genérica por laboratorios de la India a un menor costo.

Otras ideas libertarias propugnan por reafirmar los derechos individuales, cuestionar la apropiación y enfatizar en una utilización ética de los recursos, debido a su escasez para evitar conflictos entre los individuos ${ }^{32}$.

Por otro lado, el Premio Nobel de Economía, Joseph Stiglitz, propuso un sistema de premios que permitiera galardonar a aquellos que hacen innovación destacada en el campo de la industria farmacéutica, este se financiaría por un fondo común de los países de economías destacadas como el G8 y con aportes de las ONG, sector privado. Ello fortalece la equidad e incentiva la investigación, esta iniciativa fue propuesta por " $U K$ for instance, the Royal Society of arts" 33 .

Finalmente, ante la actual pandemia del coronavirus surgen las iniciativas de patent pools, propuesta efectuada por el presidente de Costa Rica, Carlos Quesada, y su ministro de Salud, Daniel Salas, en la cual se proponía unir conocimientos y toda la tecnología disponible para ser llevada a la ciencia y así\# combatir el virus, esta propuesta se basa en una economía colaborativa. Ello requerirá de un esfuerzo, con el cual los países fueran más allá\# de lo que normalmente hacen, y así poner todos los recursos y tecnología que actualmente esta\# funcionando a disposición de otros; es decir, propone dejar de lado todas las diferencias y que todos nos unamos en una "especie de refuerzo humanitario" 34 .

Además, en el evento, The WHO Covid-19 Technology Pool, el Director de South Centre, Carlos Correa, mencionó que la contribución de una ciencia es muy importante para desarrollar el eje necesario, pero hay que tener cuidado, puesto que han sido muchos los científicos seducidos por el concepto de que necesitan obtener patentes sobre lo que produzcan, así que esto se ha convertido en un patrón en muchas recetas en todo el mundo, no solo en países desarrollados sino también en países en desarrollo, y esto puede conducir a 
un paradigma diferente. Es importante destacar la ciencia abierta para que parte del mundo, en el que dicha ciencia esta\# abierta, contribuya al grupo de conocimiento para hacer realidad el acceso a las vacunas a la población al mismo tiempo, tanto países desarrollados y en desarrollo. Resalto además que es pertinente revisar las capacidades de manufactura, que son gobernadas por la oligarquía, y que es necesario el acceso al "Know how" y la cooperación para aumentar la capacidad de producción de productos que ayuden a superar la pandemia. También propuso la utilización por el gobierno de las patentes, de acuerdo con la necesidad pública $^{35}$.

\section{El análisis económico del paradigma de las patentes de medicamentos respecto de la covid-19}

La sección 8 del artículo $1^{\circ}$ de la Constitución Federal de los Estados Unidos de Norteamérica, le asigna al Congreso de la Unión la facultad de legislar y proteger los derechos de propiedad intelectual y las patentes: "a fin de promover el progreso de la ciencia y de las artes útiles otorgando, durante un tiempo limitado, a autores e inventores el derecho exclusivo a sus escritos y descubrimientos respectivos".

El órgano legislativo federal expidió en 1790 la primera ley, en materia de patentes, con el propósito de otorgar el derecho exclusivo sobre un invento, supeditado a la presentación de solicitud ante la Oficina de Patentes de los Estados Unidos, manifestando que "la creación corresponde a un proceso, máquinas, manufactura o combinación de la materia, nuevos y útiles, o [un] mejoramiento nuevo y útil de los anteriores", según lo dispuesto en el 35 U. S. Code 101.

De igual manera, la invención no tendrá que ser obvia, ostentar una utilidad práctica y no haber sido comercializada o puesta en el mercado al acceso de los consumidores con una antelación mayor a un año, con respecto a la fecha de solicitud. El solicitante que logra con éxito el registro de su invención y, en consecuencia, la obtención de la patente recibe el monopolio durante diecisiete años del uso y explotación exclusiva de esta, de manera que los terceros que deseen usarlo deberán adquirir tal derecho mediante el pago de un honorario, conocido como regalía, en virtud de una licencia de uso ${ }^{36}$.

No solo la regulación de los Estados Unidos, sino en los diferentes ordenamientos, como lo es el caso colombiano, la legislación exige que los inventores registren la creación para efectos de obtener una patente, teniendo en cuenta que dicho registro es constitutivo del derecho y, una vez obtenido, nadie podrá usar el invento sin la autorización del propietario, quien obtiene un derecho de propiedad exclusivo sobre el invento, cuyas dimensiones comprenden la duración y el alcance.

La duración comprende el número de años que deberán transcurrir a partir del momento del registro de la patente hasta su expiración. El segundo implica la cierta similitud que un producto puede guardar con una invención patentada, sin que con ello se infrinja la patente del invento original.

\section{Análisis económico del alcance de la patente farmacéutica}

Según Robert Cooter et at. ${ }^{37}$, en lo relativo al alcance del derecho de patente, cabe precisar que esta dimensión comporta patentes amplias y patentes estrechas en cuanto al manto de cobertura del derecho respecto de la naturaleza de la creación. Cuando los inventos asumen la forma de descubrimiento, al igual que sus aplicaciones, como el compuesto de una vacuna o un medicamento y la aplicación, las autoridades pueden expedir una patente dominante al descubrimiento para el pionero y una patente subordinada a la mejora.

Las patentes amplias por lo general se refieren a inventos pioneros incluyendo sus aplicaciones concretas, cuyo propósito será estimular la investigación fundamental, mientras que las patentes estrechas incentivan el 
desarrollo, que implica una serie de pequeñas mejoras y una patente por cada aplicación por separado incluso de los inventos pioneros.

A un invento pionero sigue una serie de aplicaciones, por tanto, la cuestión radica en establecer si una patente amplia, como sería la del descubrimiento pionero, se puede extender a sus aplicaciones dirigidas al desarrollo y llevarse al mercado con eficiencia económica; o si, por el contrario, las patentes estrechas serían lo más apropiado ${ }^{38}$.

En principio, no sería recibida la tesis dirigida a sustentar que si la inversión en la investigación fundamental supera la inversión en el desarrollo de aplicaciones, las patentes deberían ser amplias en su alcance, esto es abarcar además del descubrimiento fundamental la aplicación concreta o la mejora. De igual manera, tampoco es sería de recibo afirmar que si la inversión en el desarrollo de aplicaciones es superior a aquella destinada para la investigación fundamental, las patentes deberían ser estrechadas en su alcance, esto es abarcar solo la aplicación concreta o la mejora por separado, bajo la falsa premisa de que lo que finalmente se valora no es el invento pionero sino las mejoras ${ }^{39}$.

Es preciso, no perder de vista que las aplicaciones comerciales o mejoras y los descubrimientos pioneros son productos conjuntos de la investigación fundamental; dado que las aplicaciones comerciales o mejoras necesitan de la existencia de un invento pionero que dé lugar a su aparición y, a su vez, los inventos pioneros requieren de la investigación fundamental que permita su descubrimiento ${ }^{40}$.

Para que un producto conjunto, como el descrito, se pueda proveer, de manera suficiente, la compensación del proveedor debe estar por encima, al menos, de uno de los productos conjuntos, es decir, descubrimiento pionero más mejoras, pues de lo contrario el investigador fundamental no hallará incentivo suficiente para llegar a descubrimientos pioneros. De lo anterior se deduce que la investigación fundamental y el desarrollo comercial son una sola empresa desde el punto de vista económico y su remuneración deberá ser la sumatoria correspondiente al descubrimiento pionero más las mejoras conjuntamente ${ }^{41}$.

Por lo general, son unos los agentes que llevan a cabo la actividad de investigación fundamental y otros los que se ocupan del desarrollo de aplicaciones o mejoras; sin embargo, en razón a la existencia de costos de transacción que impiden a los dedicados a la investigación fundamental y al desarrollo comercial llevar a cabo negociaciones y acuerdos eficientes ${ }^{42}$. Así mismo, la problemática, en materia de incentivos, estaría superada en el escenario utópico, donde los costos de transacción serían nulos. Esto se explica, desde el teorema de Coase ${ }^{43}$, a continuación:

En ausencia de costos de transacción, las partes intervinientes en un acuerdo llegarán siempre a la solución más eficaz, con independencia de cuál sea la asignación inicial de los derechos que haya hecho el sistema jurídico, es decir que, ante la ausencia de costos de transacción, la negociación en el mercado permitirá encontrar la solución más eficiente o la que produzca mayor utilidad.

Si los costos de transacción fueran nulos, y se partiera de la racional persecución de utilidad de los agentes, estos negociarían entre sí, y aquel que le asignara una mayor utilidad al bien podría adquirirlo del otro. Dicho en otras palabras, en ausencia de problemas de información: racionalidad limitada, (entendida como cognitiva y comunicativa) oportunismo, (o conocimiento valioso que no se quiere difundir para obtener ventajas transaccionales), entre otros factores que determinan los elevados costos de transacción, los actores del mercado, en razón de una adecuada, optima y racional estimación del costo de su utilidad, a través de la negociación, determinarían la eficiencia y maximización en la distribución de los recursos, toda vez que se podría establecer quién puede obtener mayor utilidad de este y así transferirse dicho derecho eficientemente, en términos de beneficio. Según Coase, cuando el mercado, por los elevados costos de transacción, no lo puede hacer el juez debe entrar a hacerlo, con el fin de asignar eficientemente los recursos. Dicho en otras palabras, el juez debe asignar los derechos y recursos de manera beneficiosa para los involucrados, como lo haría el mercado siempre y cuando sus agentes no se extralimitaran de una racional y moderada búsqueda de utilidad. ${ }^{44}$

Del mencionado teorema, se puede establecer, en lo relativo a los inventos, que mientras los inventores no puedan entablar negociaciones sin costo alguno entre sí y celebrar contratos eficientes, el alcance de las patentes es irrelevante para la eficiencia económica. 
En respuesta a ello, y en aplicación de normativas antimonopólicas, se ha acuñado la tesis dirigida a afirmar que las patentes amplias resultan el modelo más eficiente, pues las patentes estrechas desincentivan o desalientan al investigador fundamental en sus inversiones y esfuerzos, en los casos en los que el invento pionero tiene un escaso valor por sí solo, y en los que el creador deberá ser recompensado con parte del valor de la mejora para mantener un incentivo a la generación de nuevos inventos pioneros, lo que justifica que la patente sea amplia; y, por el contrario, más estrecha para aquellos inventos o descubrimientos pioneros que tienen gran valor por sí solos, donde el pionero será recompensado de manera apropiada sin que ello implique recibir estipendios por el valor de la mejora ${ }^{45}$.

El modelo utilizado resulta apropiado, independiente de que el invento pionero tenga un escaso valor por sí solo ${ }^{46}$, como principal referente del "enfoque positivo" del análisis económico del derecho, lo cierto es que solo son patentables los inventos mientras cumplan con el requisito de altura inventiva, y, en consecuencia, el significado funcional de lo que resulta difícilmente derivable del estado de la técnica al no ser obvio, solo se puede descubrir a elevados costos; razón por la cual sería equivocado estimar una fórmula distinta a la expuesta en el párrafo anterior, donde el investigador fundamental carezca de recompensas por cuenta de un sistema de patente estrecha, puesto que la conclusión sería una aniquilación de la investigación fundamental, no solo en el ámbito de los descubrimientos farmacéuticos, sino en otras industrias.

\section{Análisis económico de la duración de la patente farmacéutica}

De acuerdo con Robert Cooter et al., ${ }^{47}$ las patentes crean un monopolio temporal de explotación sobre una creación; beneficiando al propietario e incentivando su creatividad, mientras que los precios crecen de manera proporcional al paso del tiempo que avanza, lo cual afecta la posibilidad de los consumidores para acceder a dichas invenciones y, por supuesto, desalienta la difusión de las innovaciones o descubrimientos.

La política de licenciamiento obligatorio se constituye como un remedio destinado a reducir los costos sociales de la duración excesiva de las patentes ${ }^{48}$. Esta política perfila la función social y el bien común como criterio orientador y limitante del derecho de propiedad que no puede ser ajeno a las patentes ${ }^{49} \mathrm{y}$, a su turno, permite que los interesados demanden a los poseedores de patentes ante los tribunales para que se les obligue a otorgar licencias cuando logren acreditar que sus titulares no han ejercitado su derecho a usarlas en el mercado nacional en cierto periodo, ni han licenciado su uso, siendo ello esencial, o que han ejercitado su posición de manera viciosa, abusando del monopolio de explotación obtenido, por ejemplo, restringiendo la oferta de sus inventos para afectar el precio con tendencia al alza en perjuicio de los consumidores ${ }^{50}$.

Si el demandante prueba los hechos ante el tribunal y obtiene la concesión de sus pretensiones orientadas a obligar al demandado a otorgar la licencia, la autoridad jurisdiccional estimará una regalía proporcional.

\section{La eficiencia económica y el licenciamiento obligatorio}

Como se señaló, en el artículo 31 de los $\mathrm{ADPIC}^{51}$, se establecieron flexibilidades a los derechos de los titulares propiedad intelectual, que también fueron reiteradas en la Declaración de Doha de 2001, entre ellas, se resaltan las licencias obligatorias; estas permiten limitar el monopolio de explotación de la patente, permitiendo que otro agente del mercado o al mismo Estado puedan explotarla sin autorización del titular por razones de emergencia nacional y salud pública ${ }^{52}$, pago previo de una compensación económica a favor del titular. De esta manera, los países pueden implementar las licencias obligatorias para salvaguardar la salud pública de su población ${ }^{53}$, como en el actual escenario de pandemia de coronavirus que a 9 de febrero de $2021^{54}$ ha contagiado a 107.389.998 personas a nivel mundial, ocasionando la muerte a 2.349 .171 personas. 
En el panorama regional, Brasil presenta el mayor número de contagios en América del Sur con 9.602.034 casos y 233.588 muertos. Perú tiene 1.191.291 casos y 42.237 muertos. En Ecuador se reportan 259.783 casos, de los cuales 15.086 han fallecido. Por su parte, Colombia cuenta con 2.166 .904 casos y 62.105 muertes.

De acuerdo con la jurista", "las licencias obligatorias son una de las principales 'flexibilidades' previstas en el artículo 31 del Acuerdo sobre los Aspectos de los Derechos de la Propiedad Intelectual Relacionados con el Comercio,y es uno de los instrumentos de los que disponen los gobiernos para hacer frente a problemas de salud pública" 56 .

Las licencias obligatorias no son una expropiación ${ }^{57}$ de la patente de invención, toda vez que el titular sigue siendo el mismo y tiene el derecho a recibir una remuneración adecuada ${ }^{58}$. El alcance, contenido y duración de la licencia obligatoria está determinado por el ordenamiento jurídico nacional. El titular podrá solicitar ante la autoridad nacional competente su revisión.

Sobre el particular, la OMS señala que las licencias obligatorias

permiten que el gobierno suministre la medicina en caso de abuso de derecho por parte del titular de la patente o falta de disponibilidad comercial. Estos usos deben orientarse principalmente a abastecer el mercado interno del Miembro que los autorice. $^{59}$

En Colombia, las licencias obligatorias están establecidas en la Decisión 486 del 2000 del Acuerdo de Cartagena, con la cual se establece el Régimen Común de Propiedad Intelectual. Esta norma supranacional autoriza al ejecutivo a expedir licencias obligatorias de patentes frente a la declaratoria de la existencia de razones de interés público, de emergencia o de seguridad nacional, como está señalado en el artículo 1.7, del Decreto 476 del 25 de marzo del 2020, declarando de interés en salud pública los medicamentos, vacunas y otras tecnologías en salud que sean utilizadas para el diagnóstico, prevención y tratamiento de la covid-19.

En estas circunstancias, resulta necesario examinar el impacto económico del otorgamiento masivo de licencias obligatorias sobre tecnologías patentadas o en proceso de concesión de la protección por medio de esta flexibilidad.

De acuerdo con el Grupo de Práctica de Propiedad Intelectual \& Competencia, de la firma Gómez Pinzón $^{60}$, el resultado de la experiencia de algunos países, como Brasil, Malasia, Zimbabue, Mozambique, Zambia, Ghana, Indonesia, Tailandia, Ruanda, India y Ecuador, donde los Estados han implementado tal herramienta, ha sido la promoción del acceso a los medicamentos de enfermedades tales como el VIH y el cáncer.

Según el mencionado grupo jurídico (2020), pese a que este tipo de licencias han contribuido a elevar el bienestar de la comunidad en la medida que los productos patentados son más asequibles, el impacto en términos de incentivos a la innovación y los descubrimientos pioneros en el mediano y largo plazo son dinámicos.

En este orden de ideas, por un lado, las licencias obligatorias pueden generar incentivos positivos a la innovación, toda vez que dicha innovación se forja como indispensable para efectos de poder mantener ventajas competitivas en el mercado, teniendo en cuenta que no es posible gozar de posiciones monopolísticas sostenibles ni mantener derechos de exclusividad. Por el otro, persiste el temor de los países en vía de desarrollo para implementar las licencias obligatorias, debido a la presión de las multinacionales farmacéuticas y países desarrollados. Sobre este punto, el jurista Carlos Correa $^{61}$ señala que

las experiencias de muchos países demuestran que la posible concesión de una licencia obligatoria dispara una fuerte reacción y lobbying por parte de la industria farmacéutica, como también presiones de los Gobiernos de los Estados Unidos y los países europeos.

Por otro parte, desde una perspectiva a largo plazo, las licencias obligatorias podrían afectar negativamente la efectividad esperada de los productos patentados, dada la disminución esperada en las ganancias como retorno por las inversiones en investigación y desarrollo. Lo anterior podría generar un incentivo negativo 
de abstenerse a realizar descubrimientos pioneros y aplicaciones ${ }^{62}$; empero, tampoco podría afirmarse que las patentes, al margen de licencias obligatorias, por asegurarle al titular un gran retorno económico — esto de manera proporcional-, tengan que beneficiar o promover la innovación ${ }^{63}$.

Con posterioridad a la Declaración de Doha del 2001, las licencias obligatorias se han utilizado en varias ocasiones, con el propósito de corregir ineficiencias del mercado, con resultados efectivos; por ejemplo, el Ministerio de Salud de Tailandia, en aras de proveer una cobertura universal de la atención médica, ha implementado este mecanismo dirigido a importar versiones genéricas que no se encuentran patentadas en otros países o utilizan tecnología patentada para su producción. El mencionado ministerio, en el 2006, divulgó la existencia de la primera licencia obligatoria para el antirretroviral Efavirenz, patentado por el laboratorio $\operatorname{Merck}^{64}$.

Pasados pocos meses, fueron importadas desde la India 66.000 dosis de una versión genérica y, a finales de 2007, el Estado Tailandés informó la existencia de nuevas licencias obligatorias sobre más de veinte medicamentos contra enfermedades cardiovasculares, el VIH y el cáncer, como sería el caso de Gleevec, para el tratamiento del cáncer, cuya licencia fue cancelada con posterioridad cuando Novartis, su fabricante, ofreció proporcionarlo de manera gratuita ${ }^{65}$.

En el 2008, Tailandia produjo y proporcionó una versión genérica del medicamento para el VIH denominado Kaletra del laboratorio Abbot, dejando como resultado, que estos últimos levantaran sus operaciones en Tailandia, así como la introducción de nuevos medicamentos a dicho país ${ }^{66}$.

En esta misma línea, otros países, como Brasil, han transitado una vía similar, aunque con distintos resultados. En agosto de 2001, el gobierno brasileño anunció el otorgamiento de una licencia obligatoria a un productor farmacéutico local para fabricar el antirretroviral denominado Nelfinavir, que es una versión genérica del medicamento Viracept del laboratorio Roche; empero, debido al anuncio que condujo a una serie de negociaciones, el Estado brasileño canceló la mencionada licencia obligatoria luego de que Roche acordó distribuir el medicamento en aquel país con un descuento del $40 \%$, y seis años después, con esta misma estrategia de advertir el otorgamiento de una licencia obligatoria para el uso de la patente de Merck, obtuvo otro gran descuento en el precio del antirretroviral denominado Sustiva ${ }^{67}$.

Según Stavropoulou y Valletti ${ }^{68}$, si bien esta política de descuentos expone al laboratorio titular de la patente a que se materialicen sobre sí riesgos de mercado a nivel global, como que tales precios puedan tenerse como precios de referencia en otros mercados con tendencia a la baja en sus ventas, también es cierto que les permite mantener sus cuotas de mercado en plazas como Brasil, a la vez que preservan su reputación a nivel internacional.

\section{Conclusión}

En el contexto actual de pandemia es necesario repensar el sistema de patentes de invención que se erigen como un instrumento que representa obstáculos para el acceso a los medicamentos esenciales para la población.

La eficiencia económica relativa al alcance del régimen de patentes debe entenderse de manera flexible y segmentada; por un lado, en patentes amplias y, por el otro, en patentes estrechas, en cuanto al manto de cobertura del derecho respecto de la naturaleza de la creación. Esto teniendo en cuenta que cuando los inventos asumen la forma de descubrimiento al igual que sus aplicaciones, como el compuesto de una vacuna o un medicamento y la aplicación, estamos en presencia de patentes dominantes para los descubrimientos en favor del pionero y de patentes subordinadas para las mejoras.

Por lo tanto se deberá optar por el primer mecanismo cuando el invento pionero tenga un escaso valor por sí solo, en el que se requerirá que el creador sea recompensado con parte del valor de la mejora para mantener un incentivo a la generación de nuevos inventos pioneros, lo que justifica que la patente sea amplia o dominante. O por medio del segundo mecanismo, tratándose de aquellos inventos o descubrimientos pioneros que tienen 
gran valor por sí solos, por los que el pionero será recompensado de manera apropiada sin que ello implique recibir estipendios por el valor de la mejora ${ }^{69}$.

Por otro lado, las licencias obligatorias se erigen como opciones válidas y legítimas que los Estados pueden implementar para salvaguardar la salud de sus habitantes. Dichas licencias están permitidas en normas internacionales, regionales y nacionales. A nivel regional, en Ecuador, Brasil y Chile, se ha utilizado esta institución, generando una disminución en los costos de los medicamentos.

La tendencia actual, en el escenario de discusión sobre el tema, plantea aunar esfuerzos en una economía colaborativa a través de los patent pools. A su vez, diferentes gobiernos, como Alemania, Francia, Italia y Países Bajos, se encuentran trabajando conjuntamente y celebraron un contrato con el laboratorio AstraZeneca Plc con el fin de elaborar una vacuna para combatir la covid-19 y que esta se encuentre disponible para toda Europa $^{70}$.

Además, China obtuvo la primera patente de invención de una vacuna para la covid-19 ${ }^{71}$. A su vez, el laboratorio Roche celebró un acuerdo con la farmacéutica Regeneron con el propósito de fabricar una vacuna contra la covid- $19^{72}$.

En la actualidad, existen diferentes vacunas contra la covid-19, en efecto, algunas multinacionales farmacéuticas han obtenido autorización para su comercialización ${ }^{73}$ en la Unión Europea y otras en Estados Unidos: BioNTech, Pfizer, Moderna, CureVac, AstraZeneca, Johnson \& Johnson / Janssen Pharmaceuticals, Sanofi-GSK y Sputnik V.

En cuanto a planes de vacunación contra la covid-19, a nivel mundial, se destaca Israel que ha vacunado "a más del $62 \%$ de su población" ${ }^{74}$, Reino Unido ha aplicado la vacuna al 50\% ${ }^{75}$ y Estados Unidos ha vacunado al $55 \%{ }^{76}$.

Por otra parte, existe la desigualdad y una brecha marcada entre Europa y Latinoamérica para el acceso de vacunas contra la covid-19. En efecto, mientras la Unión Europea ha vacunado cerca del $40 \%$ de sus habitantes $^{77}$, América Latina y el Caribe tan solo al 3\% de la población ha completado su esquema de vacunación contra la covid- $19^{78}$.

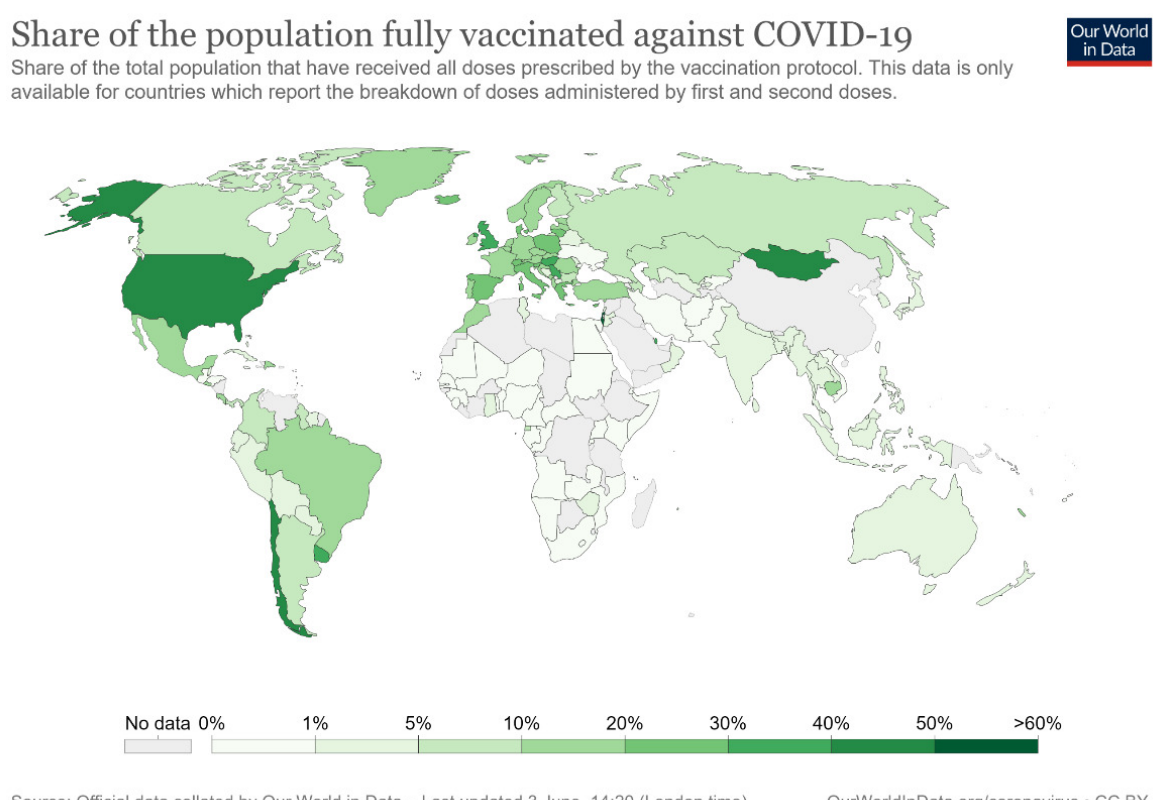

FIGURA 1.

Mapa de países con personas más vacunadas contra la covid-19

Fuente: Our World 


\section{Bibliografía}

A. Bureth et al., Le rôle du brevet dans les biotechnologies: le cas de la BioValley du Rhin Supérieur, 73 Education et formations 75 (2004).

A. Heinemann, Propiedad intelectual, Reflexiones 3-26 (2012), https://www.researchgate.net/publication/2817257 16_Le_role_du_brevet_dans_les_biotechnologies_Le_cas_de_la_BioValley_du_Rhin_Superieur

BBC New Mundo, China aprueba la primera patente de una vacuna para el coronavirus (17 de agosto de 2020), http s://www.bbc.com/mundo/noticias-53806972

C. Correa, Innovación farmacéutica, patentes incrementales y licencias obligatorias 41 (documento de investigación, 2011).

C. Correa, El uso de licencias obligatorias en América Latina (Flacso Argentina, 2018).

C. Correa, The WHO Covid-19 Technology Pool (14 de mayo de 2020). https://www.youtube.com/watch?v=iNMp $\mathrm{uQfH} 088 \& \mathrm{t}=3807 \mathrm{~s}$

C. Mascret, Licencesobligatoires demédicamentspour lespays connaissant des problèmes de santépublique: mythe ou réalité juridiques?, Médecine \& Droit 4 (2011).

C. Stavropoulou \& T. Valletti, Compulsory licensing and access to drugs, 16 The European Journal of Health Economics 83-94 (2015), https://link.springer.com/article/10.1007/s10198-013-0556-2

D. Aguirre, La patentabilidad del software y la funcio\#n social de la propiedad, Revista de Derecho, Comunicaciones y Nuevas Tecnologías (2013), https://derechoytics.uniandes.edu.co/index.php?option=com_content\&view=art icle\&id=136:la-patentabilidad-del-software-y-la-funcion-social-de-la-propiedad\&lang=es

Comisión de la Comunidad Andina Dec. 486, art. 65. Régimen común sobre propiedad Industrial (2000).

D. Encaoua, et al., Le système des brevets: idées reçues et critiques (La Documentation Française, 2012).

D. Foray, L'économiede la connaissance, La découverte (La Documentation Française, 2010).

D. Salas, The WHO Covid-19 Technology Pool, Health Action International (2020), https://www.youtube.com/watc $\mathrm{h} ? \mathrm{v}=\mathrm{iNMpuQfH} 088 \& \mathrm{t}=3807 \mathrm{~s}$

DW, ¿Cómo se vive la "nueva normalidad" en los países líderes en vacunación? (26 de abril de 2021), https://www.dw.com/es/c\%C3\%B3mo-se-vive-la-nueva-normalidad-en-los-pa\%C3\%ADses-1\%C3\%A Dderes-en-vacunaci\%C3\%B3n/a-57339963

DW, EE.UU. tiene a 100 millones de personas totalmente vacunadas contra el SARS-CoV-2 (30 de abril de 2021), https://www.dw.com/es/eeuu-tiene-a-100-millones-de-personas-totalmente-vacunadas-contra-el-sars-c ov-2/a-57393588

DW, Roche se une a laboratorio estadounidense en la carrera por una vacuna contra el covid-19 (18 de agosto de 2020), https://www.dw.com/es/roche-se-une-a-laboratorio-estadounidense-en-la-carrera-por-una-vacuna-con tra-el-covid-19/a-54620851

E. Bernal, Internet y la paradoja de la libertad (Universidad Externado de Colombia, 2019).

E. Bernal, Las repercusiones del sistema de patentes de invención en la pandemia del coronavirus (en proceso de publicación).

E. Phelps, Enrique Bour, Martin Farrell \& Horacio Piffano, Tratado de análisis económico del derecho. La Ley S.A.E. (Universidad de Buenos Aires, 2012).

E. Rengifo, Derecho de patentes (Universidad Externado de Colombia, 2016).

G. Cortés, Licencias obligatorias de patentes en épocas de pandemia (firma de abogados Gómez Pinzón, junio de 2020), https://gomezpinzon.com/licencias-obligatorias-de-patentes-en-epocas-de-pandemia/

G. Rivera, Protección de los datos de prueba farmacéuticos: aportes para una interpretación del acuerdo ADPIC favorable a lospaises en desarrollo (2014).

G. Velásquez, Pautas depatentabilidad y el acceso a medicamentos, 11 Temas de Derecho Industrial y de la Competencia 45-92 (2016). 
G. Vidaurreta, De cómo el criterio utilitarista de justificación primó en los albores del sistema de patentes: estudio de casos: Inglaterra, Estados Unidos y Francia: desde el Medioevo a la primera revolución industrial (2010) (tesis de Maestría en Propiedad Intelectual), http://ebour.com.ar/index.php?option=com_content\&task=view\&id= 240\&Itemid $=71$

I. Cárdenas, El análisis de patentes en el mundo de la inteligencia tecnológica competitiva 8 (Puzzle, 2003).

I. Vargas, Redimensión de las politicas públicas frente al acceso a medicamentos: entre la ausencia, la permisividad y el abandono estatal (Universidad del Rosario, 2015).

J. Arias. Delimitación del concepto de equidad en la Constitución Politica de 1991 Análisis de fundamentación jurisprudencial y de análisis económico del derecho, 47 Con-Texto 11-39 (2017).

J, Martínez \& F, Tripo, Innovación y propiedad intelectual: el caso de las patentes y el acceso a medicamentos (LC/MEX/ TS.2019/13) (Comisión Económica para América Latina y el Caribe [CEPAL], 2019), https://repositorio.cep al.org/bitstream/handle/11362/44744/1/S1900712_es.pdf

J. Stiglitz, Give prizes not patents 16 (New Scientist, 2006), https://www.cepal.org/es/publicaciones/44744-innovaci on-propiedad-intelectual-caso-patentes-acceso-medicamentos

L. Mumford, El mito de la máquina 9 (Emecé, 1969).

M. Alema\#n, El sistema de patentes de invencio\#n y modelos de utilidad: principios generales. Tratados administrados por la OMPI: su regulacio\#n en el acuerdo sobre los ADPIC (24 de noviembre de 2006), https://www.wipo.in t/edocs/mdocs/lac/es/ompi_oepm_oep_pi_ju_ctg_06/ompi_oepm_oep_pi_ju_ctg_06_3.pdf.

M. Bert, Brevets d'invention français (1791-1902), en Un siècle de progrès technique (1960).

M. Hardt \& A. Negri , Commonwealth: el proyecto de una revolución del común, 64, 192-203 (Ed. Akal, 2011).

M, Marzetti, Clase 3: Derecho y Economi\#a de las Patentes (Maestría en Propiedad Intelectual Flacso, 2020).

M. Weber, Économieet société (Pocket, 1971).

M. Wilson, Interpretación de las reivindicaciones en las patentes de invención, con especial referencia a las patentes farmacéuticas, Revista de Derecho 161-203 (2007), http://revistaderecho.um.edu.uy/wp-content/uploads/2012/12/Wilson-Interpretacion-de-las-reivind icaciones-en-las-patentes-de-invencion-con-especial-referencia-a-las-patentes-farmaceuticas.pdf

M. Zukerfeld, Historia de las regulaciones del acceso a los conocimientos: la propiedad intelectual en los periodos preindustrial e industrial (Maestría en Propiedad Intelectual, Flacso, 2018).

N, Harari, Sapiens: de animales a dioses: breve historia de la humanidad 33 (Debate, 2019).

NU, Comite\# de Derechos Econo\#micos, Sociales y Culturales de Naciones Unidas en la Observacio\#n General n. 14 (12 de mayo de 2000).

OMPI, China es el país que más solicitudes internacionales de patente presentó en 2019, año en que los servicios de PI, los tratados y las finanzas de la OMPI experimentaron un fuerte crecimiento (2020).

OMS, La globalización, el acuerdo sobre los ADPIC y el acceso a los productos farmacéuticos (2001).

OPS, Directora de OPS llama a cerrar las brechas de acceso a vacunas covid-19 ampliando su producción en América Latina y el Caribe (19 de mayo de 2021), https://www.paho.org/es/noticias/19-5-2021-directora-ops-llama-ce rrar-brechas-acceso-vacunas-covid-19-ampliando-su

P. Breese, Les stratégies des entreprises fondées sur la propriété intellectuelle (2010), https://www.iptrust.fr/wp-content /uploads/2016/03/Strate\%CC\%81gie_breese_masson.pdf

P. Gobert, La genese du droit de la propriete industrielle 11 (2015) (The\#se, École Doctorale de Droit, Universite\# de Bordeaux).

R. Coase et al., Le proble\#me du cou\#t social, 7, 4 Revue franc\#aise d'e\#conomie 153-193 (1992), https://www.perse e.fr/doc/rfeco_0769-0479_1992_num_7_4_1323

R. Cooter et al., Derecho y economia (Fondo de Cultura Económica, 1998).

R. Posner, El análisis económico del derecho (Fondo de Cultura Económica, 1998). 
RTVE, La vacuna en el mundo: ya se han administrado más de 2000 millones de dosis (3 de junio de 2021), https:// www.rtve.es/noticias/20210603/vacuna-coronavirus-mundo/2073422.shtml.

S. Guennif, La licence obligatoire: outil emblématique de la protection de la santépublique au Sud Revue de la régulation. Capitalisme, institutions, pouvoirs 17 (2015).

S. Kinsella, Contra a propriedade intellectual 26 (Mises Institute, 2017).

S. Lapointe, L'histoiredes brevets, 12, 3 Les Cahiers de Propriété Intellectuelle 1-23 (2000).

S. Lema, Acceso a los medicamentos: las patentes y los medicamentos genéricos: las consecuencias de considerar al medicamento como un bien de mercado y no social, 34 Rev. Bioética y Derecho (2015).

S. Montaño \& E. González, La producción y el uso del conocimiento en México y su impacto en la innovación: análisis regional de las patentes solicitadas, 22, 50 Análisis Económico 185-217 (2007).

World News, AstraZeneca agrees to supply Europe with 400 million doses of COVID-19, (13 de junio de 2020), htt ps://www.reuters.com/article/us-health-coronavirus-vaccines-idUSKBN23K0HW

\section{Notas}

* Artículo de investigación científica. El presente artículo resultado de investigación corresponde a la inquietud académica de los autores, surgida del conversatorio en línea "Patentes, Licencias Obligatorias y Covid 19", organizado y realizado el 6 de mayo del 2020 por la Maestría en Biociencias y Derecho de la Universidad Nacional de Colombia; así como del evento "IX Jornadas de Derecho Privado: Retos del Derecho Privado ante la Emergencia Sanitaria”, organizado y realizado el 13 de mayo del 2020 por la Facultad de Derecho de la Universidad Militar Nueva Granada. El proceso de investigación fue financiado con recursos propios.

1 I. Vargas, Redimensión de las politicas públicas frente al acceso a medicamentos: entre la ausencia, la permisividad y el abandono estatal, 17(01) Estudios Socio-Jurídicos 169-193 (2014), https://doi.org/10.12804/esj17.01.2014.05. S. Lema, Acceso a los medicamentos: las patentes y los medicamentos genéricos: las consecuencias de considerar al medicamento como un bien de mercado y no social, (34) Revista de Bioética y Derecho 81-89 (2015).

$2 \mathrm{Al}$ respecto, Guillermo Rivera señala que "la industria farmacéutica de investigación sostiene que en aquellas áreas no alcanzadas por las patentes de invención, el sistema de exclusividad de datos de prueba constituye el mecanismo jurídico más idóneo para asegurar el efectivo recupero de la inversión en $\mathrm{I}+\mathrm{D}$ y estimular la producción de nuevos medicamentos. En consideración de los países en desarrollo y menos desarrollados el sistema de exclusividad de datos inhibe la competencia en el mercado farmacéutico, provocando que el precio de los medicamentos se incremente por encima de lo que se alcanzaría bajo condiciones de mercado competitivo. Debe tenerse en cuenta que en estos países la mayor parte del gasto en medicamentos es afrontada por los propios pacientes". G. Rivera, Protección de los datos de prueba farmacéuticos: aportes para una interpretación del acuerdo ADPIC favorable a los paises en desarrollo (2014).

3 S. Lema, supra.

4 NU, Comite\# de Derechos Econo\#micos, Sociales y Culturales de Naciones Unidas en la Observacio\#n General n. ${ }^{\circ} 14$ (12 de mayo de 2000).

$5 \quad$ L. Mumford, El mito de la máquina 9 (Emecé, 1969).

6 N. Harari, Sapiens: de animales a dioses: breve historia de la humanidad 33 (2019).

7 S. Lapointe, L'histoire des brevets, 3 Les Cahiers de Propriété Intellectuelle 12 (2000).

8 M. Zukerfeld, Historia de las regulaciones del acceso a los conocimientos: la propiedad intelectual en los periodos preindustrial e industrial (Maestría en Propiedad Intelectual, Flacso, 2018).

9 P. Gobert, La genese du droit de la propriete industrielle 11 (2015) (the\#se, École Doctorale de Droit, Universite\# de Bordeaux).

10 El concepto de poder en Max Weber se refiere a los que tienen la oportunidad “de hacer triunfar desde una relación social su propia voluntad, aún contra las resistencias”. M. Weber, Économie et société 95 (1971). A su vez, el poder, Herrschaft —dominación-, es entendido como "la oportunidad de encontrar personas determinables dispuestas a obedecer un orden/befehl/ de contenido determinado, la obediencia pronta automática y esquematizada, en virtud de una disposición adquirida”. Id., pág. 95 (1971).

11 G. Vidaurreta, De cómo el criterio utilitarista de justificación primó en los albores del sistema de patentes: estudio de casos: Inglaterra, Estados Unidos y Francia: desde el Medioevo a la primera revolución industrial (2010) (tesis de Maestría en Propiedad Intelectual, Universidad de Buenos Aires), http://ebour.com.ar/index.php?option=com_content\&task= view\&id=240\&Itemid $=71$

12 S. Lapointe, supra nota 7. 
13 A. Heinemann, Propiedad intelectual, Reflexiones 3-26 (Universidad del Rosario, 2012).

14 M. Marzetti, Clase 3: Derecho y Economi\#a de las Patentes (Maestría en Propiedad Intelectual, Flacso, 2020).

15 A. Heinemann, supra nota 13.

16 P. Gobert, supra nota 9.

17 M. Alema\#n, El sistema de patentes de invencio\#n y modelos de utilidad: principios generales. Tratados administrados por la OMPI: su regulacio\#n en el acuerdo sobre los ADPIC (24 de noviembre de 2006), https://www.wipo.int/edocs /mdocs/lac/es/ompi_oepm_oep_pi_ju_ctg_06/ompi_oepm_oep_pi_ju_ctg_06_3.pdf

18 E. Bernal, Internet y la paradoja de la libertad (Universidad Externado de Colombia, 2019).

19 M. Bert. Brevets d'invention français (1791-1902), en Un siècle de progrès technique (1960).

20 E. Bernal, supra nota 18.

21 Id.

22 E. Rengifo, Derecho de patentes (Universidad Externado de Colombia, 2016).

23 R. Coase et al., Le proble\#me du cou\#t social, 7, 4 Revue franc\#aise d'e\#conomie 153-193 (1992).

24 E. Rengifo, supra nota 22.

25 G. Velásquez, Pautas de patentabilidad y el acceso a medicamentos, 11 Temas de derecho industrial y de la competencia 45-92 (2016).

26 E. Phelps et al., Tratado de análisis económico del derecho. La Ley S.A.E. (2012).

27 M. Hardt et at. , Commonwealth:el proyecto de una revolución del común 64 (Ed. Akal, 2011).

28 Id.

29 Id.

30 J. Martínez \& F, Tripo, Innovación y propiedad intelectual: el caso de las patentes y el acceso a medicamentos (LC/ MEX/TS.2019/13) (Comisión Económica para América Latina y el Caribe [CEPAL], 2019), https://repositorio.cep al.org/bitstream/handle/11362/44744/1/S1900712_es.pdf

31 OMPI, China es el país que más solicitudes internacionales de patente presentó en 2019, año en que los servicios de PI, los tratados y las finanzas de la OMPI experimentaron un fuerte crecimiento (2020).

32 S. Kinsella, Contra a propriedade intellectual 26 (Mises Institute, 2017).

33 J. Stiglitz, Give prizes not patents 16 (New Scientist, 2006).

34 D. Salas, The WHO Covid-19 Technology Pool (trad. propia) (Health Action International, 2020). https://www.youtu

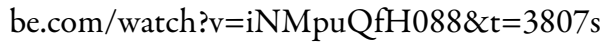

35 Id.

36 Cabe precisar que, el derecho de patente confiere a los titulares además del derecho de exclusividad dos grandes derechos reales a saber: la disposición y el uso, por consiguiente, por un lado, el propietario de la patente podrá enajenar su derecho a través de un contrato de cesión de patente, y del otro, podrá autorizar el uso, mediante un contrato de licencia de patente.

37 R. Cooter et al., Derecho y economía (Fondo de Cultura Económica, 1998).

38 Por ejemplo, una inversión de \$ 00.000 en investigación fundamental lleva al hallazgo de un descubrimiento pionero como sería el compuesto químico, que no tiene un valor comercial alguno. Luego, se realiza una inversión de $\$ \# 0.000$ en el desarrollo de una mejora cuyo valor comercial es de un millón como podría ser un antibiótico, un antiviral o una vacuna. En estas circunstancias, si se otorgan patentes amplias, el descubrimiento pionero cubrirá también la mejora; pero si, por el contrario, la ley otorga patentes estrechas, se requerirán patentes distintas para el descubridor pionero y el que hizo las mejoras.

39 Id.

40 Id.

41 Explican su afirmación trayendo a colación el caso de las ovejas que se venden por su carne y por su lana. Bajo el entendido que la carne del semoviente tiene un mayor valor comercial que la lana, si se pagare a los criadores de ovejas el valor de la lana pero no el de la carne, esto constituye un desincentivo directo para los ganaderos criadores de ovejas, por lo cual la carne y la lana son productos conjuntos de la ganadería ovina, por lo cual resulta indispensable que los criadores reciban el precio de su producto final, equivalente al valor de venta de la carne más la lana, representado en la oveja.

42 Id.

43 Id.

44 D. Aguirre, La patentabilidad del software y la funcio\#n social de la propiedad, Revista de Derecho, Comunicaciones y Nuevas Tecnologías (2013), https://derechoytics.uniandes.edu.co/index.php?option=com_content\&view=article\&id =136:la-patentabilidad-del-software-y-la-funcion-social-de-la-propiedad\&lang=es.

45 R. Cooter et al., supra nota 37.

46 En lo relativo a los elevados costos de una invención patentable, Richard Posner plantea que "si una idea que vale \$\# millón tiene un costo de descubrimiento de \$\# en lugar de \$聿50.000, la cantidad de la duplicación dispendiosa para obtener una patente será mayor, quizá \$ 49.999 mayor”. R. Posner, El análisis económico del derecho (Fondo de Cultura Económica, 1998). 
51 También están consagradas en el Convenio de París de 1883 particularmente en el Acta de la Haya de 1925 se introdujo el licenciamiento obligatorio por falta de explotación.

52 Para los efectos de este artículo solo se mencionan estas modalidades, no obstante, los ADPIC no establecieron un catálogo de modalidades de licencias obligatorias, dejando en libertad a los Estados para su regulación.

53 S. Guennif, La licence obligatoire: outil emblématique de la protection de la santé publique au Sud Revue de la régulation. Capitalisme, institutions, pouvoirs 17 (2015).

54 Covid-19 Visualizer (2020), https://www.covidvisualizer.com

55 C. Correa, Innovación farmacéutica, patentes incrementales y licencias obligatorias 41 (documento de investigación, 2011).

56 C. Correa, El uso de licencias obligatorias en América Latina (Flacso Argentina, 2018).

57 Véase Comisión de la Comunidad Andina Dec. 486, art. 65. Régimen común sobre propiedad Industrial (2000).

58 Para efectos de fijar la remuneración, se tendrá en cuenta "el contexto, el valor económico de la utilización autorizada en el país importador, las circunstancias humanitarias o no comerciales". C. Mascret, Licences obligatoires de médicaments pour les pays connaissant des problèmes de santé publique: mythe ou réalité juridiques?, Médecine \& droit 4 (2011).

59 OMS, La globalización, el acuerdo sobre los ADPIC y el acceso a los productos farmacéuticos (2001).

60 G. Cortés, Licencias obligatorias de patentes en épocas de pandemia (firma de abogados Gómez Pinzón, junio de 2020). https://gomezpinzon.com/licencias-obligatorias-de-patentes-en-epocas-de-pandemia/

61 C. Correa, supra nota 55.

62 G. Cortés, supra nota 60.

63 Merece especial atención la falta de trasparencia en los costos de investigación y desarrollo de medicamentos, al respecto, el economista Germa\#n Vela\#squez menciona el artículo, An Innovative Approach to R\&D for Neglected Patients: Ten Years of Experience and Lessons Learned by DNDi, publicado en la revista BioSocieties, en la cual argumenta que el costo real de la I+D es, de hecho, una fraccio\#n de las comúnmente citadas estimaciones. De acuerdo con los autores Light y Warburton, el costo medio de la I+D para desarrollar un medicamento vari\#a entre 13 y 204 millones de do\#lares, dependiendo del tipo de producto. Los autores estiman un costo medio de 43,4 millones de do\#lares para la I+D de cada nueva droga. Y concluyen: "esto esta\# muy lejos de los 802 millones o 1,3 billones de do\#lares reclamados por la industria".

64 C. Stavropoulou \& T. Valletti, Compulsory licensing and access to drugs, 16 The European Journal of Health Economics 83-94 (2015), https://www.proquest.com/docview/1643105576;

65 Id.

66 Id.

$67 \mathrm{Id}$.

$68 \mathrm{Id}$.

69 R. Cooter et al., supra nota 37.

70 World News, AstraZeneca agrees to supply Europe with 400 million doses of COVID-19 (13 de junio de 2020), https ://www.reuters.com/article/us-health-coronavirus-vaccines-idUSKBN23K0HW

71 BBC New Mundo, China aprueba la primera patente de una vacuna para el coronavirus (17 de agosto de 2020), https ://www.bbc.com/mundo/noticias-53806972

72 DW, Roche se une a laboratorio estadounidense en la carrera por una vacuna contra el COVID-19 (18 de agosto de 2020), https://www.dw.com/es/roche-se-une-a-laboratorio-estadounidense-en-la-carrera-por-una-vacuna-contra-e 1-covid-19/a-54620851

73 E. Bernal, Las repercusiones del sistema de patentes de invención en la pandemia del coronavirus (en proceso de publicación).

74 DW, ¿Cómo se vive la “nueva normalidad” en los países líderes en vacunación? (26 de abril de 2021), https://www.dw.com/es/c\%C3\%B3mo-se-vive-la-nueva-normalidad-en-los-pa\%C3\%ADses-1\%C3\%ADderesen-vacunaci\%C3\%B3n/a-57339963;

75 Id.

76 DW, EE.UU. tiene a 100 millones de personas totalmente vacunadas contra el SARS-CoV-2 (30 de abril de 2021), http s://www.dw.com/es/eeuu-tiene-a-100-millones-de-personas-totalmente-vacunadas-contra-el-sars-cov-2/a-57393588

77 RTVE, La vacuna en el mundo: ya se han administrado más de 2000 millones de dosis (3 de junio de 2021), https://w ww.rtve.es/noticias/20210603/vacuna-coronavirus-mundo/2073422.shtml 
78 OPS, Directora de OPS llama a cerrar las brechas de acceso a vacunas COVID-19 ampliando su producción en América Latina y el Caribe (19 de mayo de 2021), https://www.paho.org/es/noticias/19-5-2021-directora-ops-llama-cerrar-br echas-acceso-vacunas-covid-19-ampliando-su

\section{Licencia Creative Commons CC BY 4.0}

Cómo citar este artículo: Edwin Jesith Bernal Ramírez \& David Andrés Aguirre Soriano, Las patentes farmacéuticas en razón de la covid-19 desde la perspectiva del Law and economics movement, 70 Vniversitas (2021), https://doi.org/10.11144/Javeriana.vj70.pfrc 